\title{
High-sensitivity mapping of ammonia emission in the Trumpler 14/Car I photodissociation region
}

\author{
T. Young, ${ }^{1 \star}{ }^{\star}$ S. Horiuchi, ${ }^{2}$ J. A. Green ${ }^{3}$ and H. Jerjen ${ }^{1}$ \\ ${ }^{1}$ Research School of Astronomy and Astrophysics, The Australian National University, Mt Stromlo Observatory, via Cotter Rd, Weston, ACT 2611, Australia \\ ${ }^{2}$ CSIRO Astronomy and Space Science, Canberra Deep Space Communications Complex, PO Box 1035, Tuggeranong, ACT 2901, Australia \\ ${ }^{3}$ CSIRO Astronomy and Space Science, Australia Telescope National Facility, PO BOX 76, Epping, NSW 1710, Australia
}

Accepted 2013 August 15. Received 2013 August 5; in original form 2012 August 18

\begin{abstract}
The photodissociation region in the immediate vicinity of the Trumpler 14 star cluster represents the nearest source $(2.3 \mathrm{kpc})$ to study the physical parameters of a photodissociation region due to feedback from a large population of O-type stars. It resides in the Carina Nebula, a region rich in high-mass stars. We present the first detection and maps of the ammonia inversion emission of the first three metastable lines, $(J, K)=(1,1),(2,2)$ and $(3,3)$, in the Carina I dark cloud. These observations, made with the Tidbinbilla $70 \mathrm{~m}$ radio telescope, are the initial results of a programme to implement full mapping capability. We also present velocity maps together with the optical depth and rotational $\left(R_{21}\right)$ temperatures. The derived linewidths and rotational temperatures support evidence for an ionization front associated with the Carina I-E and Carina I-W substructures. Detection of the $(3,3)$ metastable line suggests significantly higher kinetic temperatures in the molecular clumps than those derived with $\mathrm{CO}$ studies, implying that the $\mathrm{CO}$ emission is saturated despite photodissociation. The kinematics of the Carina I-E and Carina I-W ammonia substructures are consistent with studies of radio recombination and molecular lines. The prevailing morphological paradigm for Car I is considered, particularly by comparing the molecular structure traced by the ammonia emission to stellar infrared and radio sources in the region.
\end{abstract}

Key words: ISM: clouds - photodissociation region (PDR) - galaxies: star formation.

\section{INTRODUCTION}

As the largest and brightest nebula in the Southern hemisphere, renowned for its concentration of high-mass stars, the molecular environment of the Carina Nebula has been the subject of several comprehensive studies (e.g. Brooks, Whiteoak \& Storey 1998; Brooks, Storey \& Whiteoak 2001; Brooks et al. 2003; Tapia et al. 2003, 2006; Rathborne et al. 2004; Smith \& Brooks 2008, and references therein). Of particular interest are the feedback triggered star formation processes occurring within Carina I's associated dark clouds (e.g. de Graauw et al. 1981; Whiteoak \& Otrupcek 1984; Brooks et al. 1998; Zhang et al. 2001) and their associated photodissociation regions (PDR). The Trumpler 14 (Tr 14) and Trumpler 16 star clusters combined contain over 30 O stars, which contribute significantly to the energy input of the region (Feinstein 1995; Vazquez et al. 1996). At a short distance of only $2.3 \mathrm{kpc}$ (Hur, Sung \& Bessell 2012), the Tr 14 cluster represents an excellent opportunity to study the molecular properties of a PDR as extreme as the 30 Doradus star-forming region of the Large

^E-mail: tyoung@mso.anu.edu.au
Magellanic Cloud (see de Marchi et al. 2011 for a recent study on 30 Doradus).

The structure of the Carina I dark cloud has been described extensively in the literature by the aforementioned studies. The radio recombination (H110 $\alpha$ ) study of Brooks et al. (2001) finds ionizing fluxes within the greater molecular cloud consistent with the expected photodissociation fronts originating from the $\operatorname{Tr} 14$ cluster. Two emission structures are imaged, Car I-W and Car I-E, which have radial velocities of -19 and $-25 \mathrm{~km} \mathrm{~s}^{-1}$, respectively. The detection of two compact $\mathrm{H}$ II regions provides the first evidence for triggered star formation in Carina I. Rathborne et al. (2002) later confirm this structural model by observing the emission of polycyclic aromatic hydrocarbons directly tracing the photodissociation fronts and similarly Brooks et al. (2003) confirm this with observations of CO and CS emission. The infrared studies of Tapia et al. $(2003,2006)$ detected stellar sources at the line of sight of the interface between Car I-E and Car I-W, many of which exhibit either or both near- and mid-infrared excesses. Taken together, these studies describe a nearly complete morphology tracing the stellar, diffuse molecular and ionized components of the Car I dark cloud; whereby, an embedded young cluster of stars is driving the ionization and outflow of the surrounding molecular gas in 
addition to the ultraviolet feedback from the centre of the $\operatorname{Tr} 14$ cluster.

Related studies (Smith et al. 2000; Brooks et al. 2001) have shown evidence for high-mass star formation in the region. Despite the molecular emission of ammonia serving as a tracer of highdensity clumps and thus typically correlating with local regions of star formation, Car I has not, until this study, had ammonia emission detected. The only detection of ammonia in the region to date was reported in the study of Smith et al. (2006), detecting emission towards Eta Carinae. In the present study, utilizing the excellent sensitivity of the $70 \mathrm{~m}$ antenna at the Canberra Deep Space Communication Complex (which is located in Tidbinbilla and operated by CSIRO for NASA), we present not only a detection of ammonia, but also partially sampled emission map and velocity maps along with derivation of optical depths and rotational temperatures.

Although physical parameters can be determined from CO emission, such as those derived in the study of Smith \& Brooks (2008), the ammonia to molecular hydrogen abundance is significantly lower relative to $\mathrm{CO}$ and is unaffected by depletion at lower temperatures due to freezing out (Bergin \& Langer 1997). Furthermore, CO quickly saturates in dense environments due to its relatively high abundance (Pineda, Caselli \& Goodman 2008), whereas ammonia is typically found only in densities greater than $10^{4}$ molecules per cubic centimetre (Stahler \& Palla 2005). Observations of ammonia emission in the millimetre regimes are thus complementary to existing $\mathrm{CO}$ emission observations.

Given that ammonia traces dense molecular environments, it provides a unique opportunity to sample the kinematics of dense molecular cores surrounding the embedded young stellar objects and ionized interfaces to a greater depth where sufficient resolution can be achieved. Although the resolution of the Tidbinbilla dish at $22 \mathrm{GHz}$ is only $\sim 40$ arcsec, its excellent sensitivity means that if ammonia emission is present, its detection could be quickly achieved. Such a detection could provide the basis for follow up with higher resolution observations.

This paper is structured such that Section 2 describes the observations and newly implemented data reduction pipeline which was used to detect and map the molecular emission of ammonia; Section 3 presents maps of the ammonia emission and derived parameters and Section 4 discusses the implications of the molecular properties that we determine.

\section{OBSERVATIONS AND ANALYSIS}

\subsection{Observations}

The observations of Car I were conducted using the Tidbinbilla $70 \mathrm{~m}$ radio telescope in 2011 January on the dates of 16, 21, 24 and 26, February 3 and August 11. The observations were made to construct a map of the $\mathrm{NH}_{3}(1,1),(2,2)$ and $(3,3)$ transitions (see Table 1 for rest frequencies and inversion energies), with a 10 by 5 grid of pointings spaced at 30 arcsec, undersampling the primary beam of $\sim 40$ arcsec. The pointing spacing was restricted by telescope time constraints, but has been accounted for in the data reduction and gridding (see Section 2.2). This strategy mapped out a 4.5 arcmin by 2.5 arcmin region in RA and Dec., respectively, centred at a right ascension of $10^{\mathrm{h}} 22^{\mathrm{m}} 00^{\mathrm{s}}$ and declination of $-59^{\circ} 34^{\prime} 30^{\prime \prime}$ (J2000). This particular mapping strategy was chosen based on pilot observations centred at the peak emission of $\mathrm{CO}$ in Smith \& Brooks (2008) conducted late in 2010 aimed at detecting ammonia in the region.
Table 1. The frequencies and energies of the first three metastable states of ammonia (Townes \& Schawlow 1955).

\begin{tabular}{cccc}
\hline Transition $^{a}$ & $f(\mathrm{GHz})$ & $E_{\mathrm{l}}(\mathrm{K})^{b}$ & $E_{\mathrm{u}}(\mathrm{K})^{c}$ \\
\hline$(1,1) \mathrm{u} \rightarrow(1,1) 1$ & 23.69450 & 22.13 & 23.26 \\
$(2,2) \mathrm{u} \rightarrow(2,2) 1$ & 23.72265 & 63.31 & 64.45 \\
$(3,3) \mathrm{u} \rightarrow(3,3) 1$ & 23.87013 & 122.39 & 123.54
\end{tabular}

${ }^{a}$ The inversion transition from upper to lower state.

${ }^{b}$ The lower state energy in kelvin above the ground state. ${ }^{c}$ The upper state energy in kelvin above the ground state.

The observations were conducted in fair weather conditions, with system temperatures in the range of 40-120 K. Two intermediate frequency (IF) bands were observed with a bandwidth of 2048 channels across $64 \mathrm{MHz}$ corresponding to a channel width of $31.25 \mathrm{KHz}$ (a velocity channel width of $\sim 0.4 \mathrm{~km} \mathrm{~s}^{-1}$ ). The first IF was set to an observational frequency of $23708.807 \mathrm{MHz}$ and the second to $23870.000 \mathrm{MHz}$, so that the $\mathrm{NH}_{3}(1,1)$ and $(2,2)$ inversion transitions were simultaneously observable in the first band and the $(3,3)$ transition in the second band. At the beginning of each observing session, the pointing errors were corrected by observing the quasar QSO B1104-445, $16^{\circ}$ offset from the target. The pointing accuracy is expected to be $\leq 2$ arcsec. A main beam efficiency factor $\left(n_{\mathrm{mb}}\right)$ of 0.48 was adopted in correcting the observed temperatures, as measured by Greenhill et al. (2003). An integration time of 16 min per point, including $8 \mathrm{~min}$ on source and $8 \mathrm{~min}$ off source, was adopted. The total observing time, including overheads, amounted to $15 \mathrm{~h}$. The resulting median signal $\mathrm{rms}$ is $0.08 \mathrm{~K}$ for the reduced spectra ( $T_{\mathrm{mb}}$ scale).

In order to verify the optical depths and rotational temperatures derived with the mapping observation (discussed in the following section), a separate deep observation was conducted on the August 26 and centred on right ascension $10^{\mathrm{h}} 43^{\mathrm{m}} 20^{\mathrm{s}}$ and declination $-59^{\circ} 34^{\prime} 30^{\prime \prime}$ (J2000). This corresponded to a bright region of emission with a radial velocity of $-20 \mathrm{~km} \mathrm{~s}^{-1}$ (Car I-W). With an integration time of $1 \mathrm{~h}$, we were able to obtain greater than $3 \sigma$ detections for the satellite hyperfine components. These were used to independently derive parameters, ensuring that results were consistent. Comparatively, the mapping observations typically produced a $2 \sigma$ detection or less in all but the central bright regions. Unfortunately, due to time constraints, we were unable to perform a deep observation of Car I-E [emission peak with a radial velocity ( $\left.V_{\mathrm{LSRK}}\right)$ of $-23 \mathrm{~km} \mathrm{~s}^{-1}$ located at right ascension $10^{\mathrm{h}} 43^{\mathrm{m}} 20^{\mathrm{s}}$ and declination $-59^{\circ} 34^{\prime} 30^{\prime \prime}$ ].

\subsection{Analysis}

We implemented a new data reduction pipeline to produce maps of the molecular emission observed with the Tidbinbilla radio telescope. The pipeline was designed to take advantage of three preestablished Australia Telescope National Facility (ATNF) reduction packages: (1) the ATNF spectral analysis package $\mathrm{ASAP}^{1}$, which was designed with the primary role of reducing pointed observation data from the Mopra and Tidbinbilla radio telescopes, performing baseline subtraction and spectral calibration; (2) GRIDZILLA, part of

\footnotetext{
${ }^{1}$ Documentation on ASAP can be found from http://svn.atnf.csiro.au/ trac/asap.
} 
the combined LIVEDATA and GRIDZILLA packages ${ }^{2}$ used for on-the-fly mapping observations with Mopra and the Parkes Radio telescope, performing spatial regridding of spectral line data into spectral data cubes and (3) MIRIAD (Sault, Teuben \& Wright 1995), which contains a range of calibration and reduction features suitable for the Australia Telescope Compact Array including moment analysis suitable for emission mapping. The full details of the combined pipeline, wrapped within a Python script, are given below.

(i) Reduction of raw data with the ASAP package. Quotient spectra, continuum subtraction, elevation and atmospheric calibrations (by applying a gain curve and measuring the opacity at each observation session by tipping the antenna from horizon to zenith, respectively) are performed for every map point in the data set. The reduced points are then saved to the single dish fits (SDFITS) format aligning the frequency to the first point in the observation.

(ii) Spatial gridding with GRIDZILLA. A point spread function full width half-maximum and cut-off radius are used when interpolating to the user-defined pixel size. In our observations, we smoothed the data to 1 arcmin and gridded with 30 arcsec pixels ensuring Nyquist sampling. The output results in a spectral cube fits file.

(iii) Integrated intensity, peak intensity, velocity maps, optical depths and rotational temperatures are generated from the spectral cubes using the MIRIAD MOMENT and ODNH3 tasks.

(iv) All final map images are produced using the APLPY (Astronomical Plotting Library in Python) package.

\section{RESULTS}

In this section, emission maps of the integrated source temperature $\left(T_{\mathrm{mb}}\right.$, corrected for atmospheric attenuation and aperture efficiency as discussed in Section 2.1) and velocity maps of Car I are presented along with Gaussian-fitted emission spectra from the deep observation and the associated fit and derived parameters. All derived parameters are calculated using the standard analytical interpretation of ammonia emission, based on the discussions of Ho et al. (1977), Townes \& Ho (1983), Stutzki \& Winnewisser (1985) and Ungerechts, Winnewisser \& Walmsley (1986). As a matter of convenience, inversion emission from the first, second and third metastable levels of ammonia, may be referred to by the $(J, K)$ levels transitions of $(1,1),(2,2)$ and $(3,3)$, respectively, as shorthand. Two distinct clouds of ammonia are detected along declination $-59^{\circ} 34^{\prime} 30^{\prime \prime}$ within the mapped field of view. The cloud centred at right ascension $10^{\mathrm{h}} 43^{\mathrm{m}} 20^{\mathrm{s}}$ has a peak radial velocity $\left(V_{\mathrm{LSRK}}\right)$ of $-23 \mathrm{~km} \mathrm{~s}^{-1}$ (hereafter Car I-E) and the cloud at $10^{\mathrm{h}} 43^{\mathrm{m}} 30^{\mathrm{s}} .0$ has a radial velocity of $-20 \mathrm{~km} \mathrm{~s}^{-1}$ (hereafter Car I-W).

\subsection{Emission maps}

For the Car I observation, emission spectra were obtained which enabled us to measure the radial velocities, peak and integrated line temperatures. Emission maps of the $\mathrm{NH}_{3}(1,1),(2,2)$ and $(3,3)$ levels were then derived (Fig. 1). The integrated intensity emission maps are sliced in velocity to best illustrate the two distinct clouds of ammonia. For the $(1,1)$ transition, these clouds are at a declination of $-59^{\circ} 34^{\prime} 00^{\prime \prime}$, with the brightest cloud centred at right ascension of $10^{\mathrm{h}} 43^{\mathrm{m}} 20^{\mathrm{s}}$ and the other cloud at $10^{\mathrm{h}} 43^{\mathrm{m}} 30^{\mathrm{s}} .0$. In analysing the peak moment maps, the emission intensity decreases with increasing

\footnotetext{
2 The GRIDZILLA/LIVEDATA distribution can be found from http://www. atnf.csiro.au/people/mcalabre/livedata.html.
}

metastable level, but there is still a significant amount of $(2,2)$ and $(3,3)$ emission.

The presence of the $(3,3)$ emission indicates that temperatures are significantly higher than would be expected for an infrared dark cloud (IRDC; Fontani et al. 2012). Another interesting trend in the peak emission maps is that the eastern cloud shifts further to the east for the $(2,2)$ and $(3,3)$ transitions relative to the $(1,1)$ transition and similarly for the western cloud, which shifts further to the west. This is indicative of a radial temperature gradient across the map, with higher temperatures to the fringes of the mapped region (discussed further in Section 4). The velocities associated with each cloud are very distinct, with little deviation across the mean value within each cloud. There is however an increase in velocity dispersion in Car I-E and Car I-W towards their respective ionization front.

The overlaid markers in Fig. 1 demonstrate the complex morphology of the region. Stellar sources (indicated by stars, Fig. 1) lie along the line-of-sight interface between the Car I-E and Car I-W molecular clouds and are closest to the Car I-W H110 $\alpha$ radio-continuum sources (squares). In close proximity to all three radio-continuum sources is an associated $\mathrm{H}$ II region. The $\mathrm{H} 2 \mathrm{O} 287.37-00.62$ maser source (indicated by the circle) discovered by Caswell et al. (1989) lies along the direct line of sight of the Car I-E emission, whilst extending westwards are a series of mid-infrared molecular and recombination emission knots (indicated by the pentagons).

\subsection{Velocity maps and derived parameters}

In Fig. 2, we present the velocity maps derived using the newly implemented reduction pipeline. For the central region along declination $-59^{\circ} 34^{\prime} 15^{\prime \prime}$, signal-to-noise ratio was sufficient such that optical depth and rotational temperature calculations could be carried out using the standard analytical interpretation of ammonia emission (i.e. manual determination through peak fitting and use of the analytical formula from Townes \& Ho 1983). These calculations are presented in Table 2.

\subsection{Deep observation}

Emission spectra of the Car I-W peak obtained with the deep observation discussed in Section 2.1 are presented in Fig. 3. The overlaid Gaussian-fitted parameters are presented in Table 3. The following parameters are derived from the Gaussian fits with associated Gaussian uncertainties: the optical depth $\tau_{(1,1, m)}=1.2 \pm 0.2$ and the rotational temperature $T_{\mathrm{R}}^{21}=21.9 \pm 3.6 \mathrm{~K}$. Large wings are visible around the main peak. Unfortunately, the signal-to-noise ratio in the spectral cube is not sufficient to identify these at other positions, and so we are unable to conclude with great certainty its origin. As evident in Fig. 3, this has not affected the fit. Given that Car I-W contains an embedded young stellar population (see Sections 3.1 and 4.2 for further discussion), it is possible that this could be the product of an outflow.

\section{DISCUSSION}

In contrast to the nearby and immensely studied Orion nebula, Car I is poorly studied for ammonia. The only previous study which has successfully observed ammonia in the vicinity that of Smith et al. (2006) detected the ammonia $(J, K)=(3,3)$ transition towards the luminous blue variable star Eta Carinae. The observations presented in this report are the first comprehensive study of ammonia emission in Car I and indeed that of a PDR, observing the PDR associated with the $\operatorname{Tr} 14$ cluster. This region has been examined in detail in $\mathrm{CO}$ 

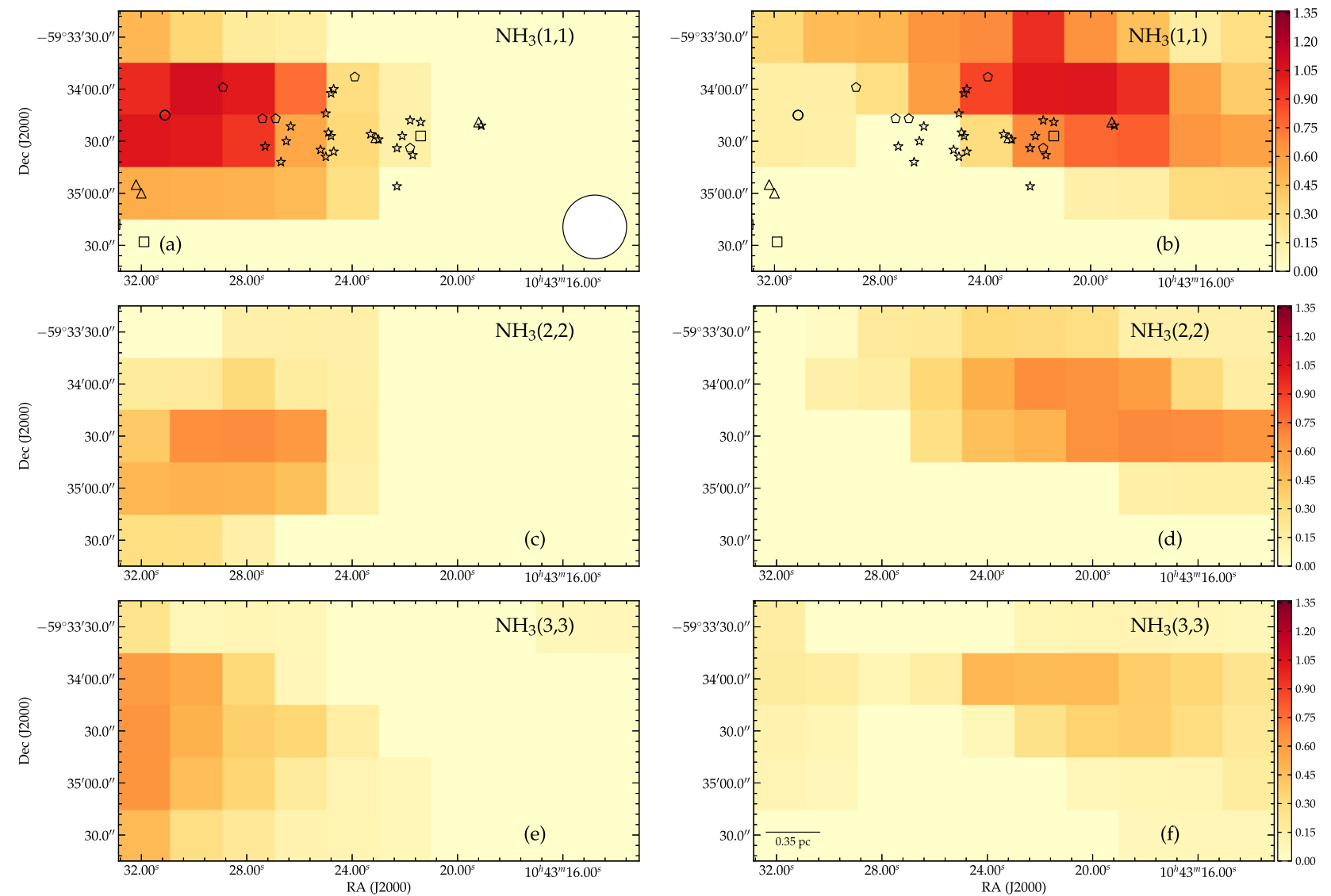

Figure 1. The integrated main beam $\left(T_{\mathrm{mb}}\right)$ intensity $\left(\mathrm{K} \mathrm{km} \mathrm{s}^{-1}\right)$ maps for the first, second and third (top to bottom, respectively) metastable transitions of ammonia in Car I. The horizontal axis shows the right ascension coordinate and the vertical axis shows the declination coordinate. The yellow-orange-red colour scale represents the integrated intensity of the main hyperfine at the gridded pixel. The left and right columns denoted by (a) and (b) for the first metastable level, (c) and (d) for the second, and (e) and (f) for the third are slices over the velocity ranges -21 to $-25 \mathrm{~km} \mathrm{~s}^{-1}$ (left) and -17 to $-21 \mathrm{~km} \mathrm{~s}{ }^{-1}$ (right), respectively. The primary beam size, scale bar and colour scale (applicable to both left and right image columns) are indicated by the black outlined circle in panel (a), the scale bar in the bottom left of panel (f) and the colour bar on the right image column, respectively. Markers are overlaid on panels (a) and (b) indicating the following. Stellar near-infrared sources (stars; Tapia et al. 2003, 2006); mid-infrared molecular and recombination emission knots (pentagons; Tapia et al. 2006); H II regions (triangles; Gardner et al. 1970; Kuchar \& Clark 1997; Giveon et al. 2002); the H2O 287.37-00.62 maser source (circle; Caswell et al. 1989) and H110 $\alpha$ radio-continuum sources (squares; Brooks et al. 2001).
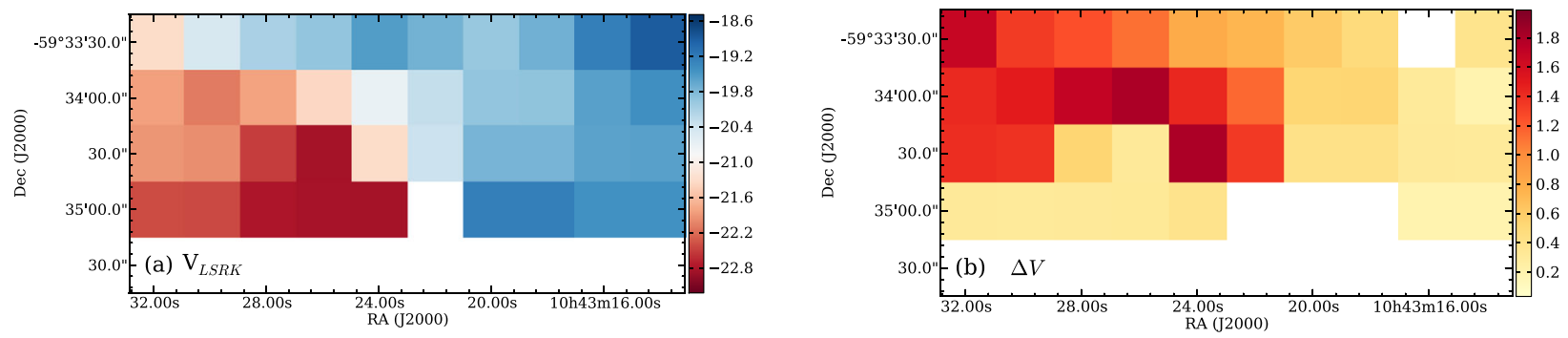

Figure 2. First and second moment maps of Car I. The horizontal axis shows the right ascension coordinate and the vertical axis shows the declination coordinate. (a) $V_{\mathrm{LSRK}}$, the measured line velocity for the first metastable transition $\left(\mathrm{km} \mathrm{s}^{-1}\right)$. The red-white-blue colour scale and its associated colour bar (located right of the panel) represent the line velocity at the gridded pixel. (b) $\Delta V$, the velocity dispersion map ( $\mathrm{km} \mathrm{s}^{-1}$ ). The yellow-orange-red colour scale and its associated colour bar (located right of the panel) represent the amount of line dispersion at the gridded pixel.

and CS molecular emission in the study of Brooks et al. (2003), and the parameters derived with these molecules provide a comparison to those in this report.

The emission maps presented in fig. 7 of Brooks et al. (2003) also demonstrate the Car I-E and Car I-W velocity substructures (in addition to other structures). Although the spatial structure of the $\mathrm{CO}$ and CS emission is remarkably similar when compared to the inte- grated intensity maps presented here; fig. 6 of Brooks et al. (2003) shows comparatively higher velocity dispersion for the $\mathrm{CO}(2-1)$ emission. Comparisons of ammonia and $\mathrm{CO}$ emission maps have demonstrated this phenomena in the past. The proposed schematic of Orion Molecular Cloud 1 in Batrla et al. (1983) is one such example. Typically, the ammonia to molecular hydrogen abundance is significantly lower relative to $\mathrm{CO}$ in these types of regions and is 
Table 2. The derived rotational temperature and optical depth values along the central region of emission (declination $-59^{\circ} 34^{\prime} 15^{\prime \prime}$ ) where signal-to-noise ratio was sufficient to allow such a calculation.

\begin{tabular}{ccc}
\hline $\begin{array}{c}\text { Right ascension } \\
(\mathrm{h} \mathrm{m} \mathrm{s})\end{array}$ & $\begin{array}{c}T_{\mathrm{R}}^{21} \\
(\mathrm{~K})\end{array}$ & $\tau_{(1,1, m)}$ \\
\hline 104330 & 27 & 1.8 \\
104327 & 25 & 1.9 \\
104325 & 22 & 1.9 \\
104322 & 22 & 1.9 \\
104320 & 22 & 1.7 \\
104318 & 22 & 1.6 \\
104315 & 23 & 1.3 \\
104313 & 23 & 1.6 \\
\hline
\end{tabular}

unaffected by depletion at lower temperatures due to 'freezing out' (Bergin \& Langer 1997) or saturation in higher densities. As such, Car I also demonstrates that ammonia is an excellent complimentary tracer of higher density gas, found when densities are greater than $10^{4}$ molecules per cubic centimetre (Stahler \& Palla 2005).

\subsection{Physical parameters}

The line velocity and velocity dispersion maps (Fig. 2) clearly delineate two separate velocity structures, Car I-E at $-23 \mathrm{~km} \mathrm{~s}^{-1}$ and Car I-W at $-20 \mathrm{~km} \mathrm{~s}^{-1}$. In Car I-W and Car I-E, the velocity dispersion increases from $0.2 \mathrm{~km} \mathrm{~s}^{-1}$ in the western and extents up to as high as $1.75 \mathrm{~km} \mathrm{~s}^{-1}$ in the east (see Fig. 2b), which is consistent with the location of the ionization fronts. However, for Car I-W, the increased dispersion also corresponds to the line-of-sight region of overlap between the two clouds; and this could possibly be a product of the blended velocities.

Car I demonstrated an 'upward slope' trend across the peaks of the hyperfine components (Fig. 3). This occurs because for most pixels there was a small systematic deviation from local thermodynamic
Table 3. The fitted Gaussian parameters to the spectral lines in the core observation of the Carina nebula. $T_{\text {peak }}$ is the observed peak main beam temperature, $V_{\mathrm{LSRK}}$ is the velocity at the local standard of rest frequency and $\Delta V$ is the linewidth of the measured peak (the uncertainties include the channel response).

\begin{tabular}{cccc}
\hline \multicolumn{1}{c}{ Transition } & $\begin{array}{c}T_{\text {peak }} \\
(\mathrm{K})\end{array}$ & $\begin{array}{c}V_{\text {LSRK }} \\
\left(\mathrm{km} \mathrm{s}^{-1}\right)\end{array}$ & $\begin{array}{c}\Delta V \\
\left(\mathrm{~km} \mathrm{~s}^{-1}\right)\end{array}$ \\
\hline$(1,1$, left outer $)$ & $0.23 \pm 0.03$ & $-39.4 \pm 0.1$ & $1.8 \pm 0.3$ \\
$(1,1$, left inner $)$ & $0.38 \pm 0.03$ & $-27.47 \pm 0.06$ & $1.9 \pm 0.2$ \\
$(1,1$, main $)$ & $0.93 \pm 0.03$ & $-19.90 \pm 0.02$ & $1.8 \pm 0.2$ \\
$(1,1$, right inner $)$ & $0.37 \pm 0.03$ & $-12.36 \pm 0.06$ & $1.5 \pm 0.2$ \\
$(1,1$, right outer $)$ & $0.40 \pm 0.03$ & $-0.43 \pm 0.06$ & $1.8 \pm 0.2$ \\
$(2,2$, left outer $)$ & $0.05 \pm 0.03$ & $-46.4 \pm 0.8$ & $2 \pm 2$ \\
$(2,2$, left inner $)$ & $0.06 \pm 0.04$ & $-36.2 \pm 0.5$ & $1.5 \pm 1$ \\
$(2,2$, main $)$ & $0.62 \pm 0.03$ & $-19.90 \pm 0.05$ & $1.9 \pm 0.2$ \\
$(2,2$, right inner $)$ & $0.07 \pm 0.04$ & $-3.6 \pm 0.4$ & $1.5 \pm 1$ \\
$(2,2$, right outer $)$ & $0.06 \pm 0.03$ & $-5.8 \pm 0.7$ & $2.5 \pm 2.5$ \\
\hline
\end{tabular}

equilibrium (LTE) for the outermost hyperfine components: meaning essentially the highest and lowest $V_{\text {LSRK }}$ hyperfine components had too strong and too weak brightness temperatures, respectively, to what would be typically expected. A physical explanation of this systematic deviation involves photon trapping for the lower levels as discussed in Matsakis et al. (1977). This had little impact on the derivation of the optical depth, particularly, since this effect is only pronounced on the outer hyperfines. Each pair of hyperfine ratios (the left and right inner pairs and the left and right outer pairs) were averaged before solving for optical depth. Since the photon trapping would increase the left ratio and decrease the right ratio, by averaging over the ratios (e.g. the left outer hyperfine and the right outer hyperfine) before solving for optical depth, we essentially mitigated the trapping effect if not cancelling it completely. This would yield two optical depth values, one for the inner pair and one for the outer pair and would always agree within uncertainty margins. The quoted optical depths within this study are derived from the sigma-weighted mean of these values.

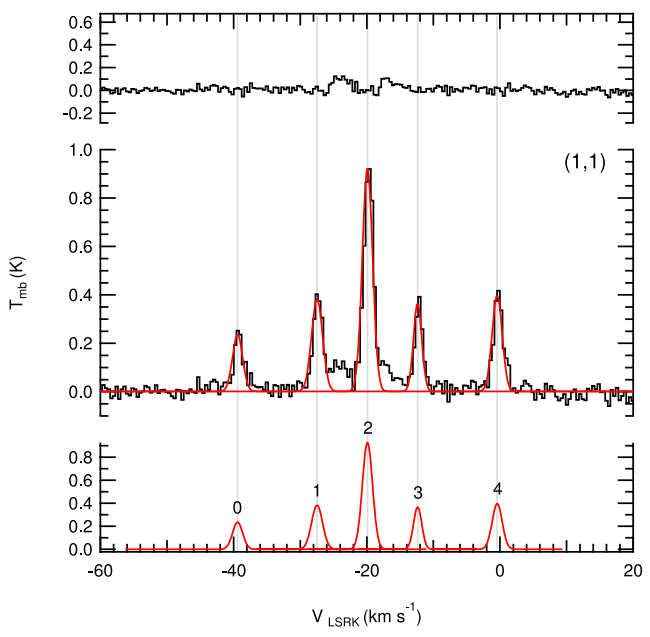

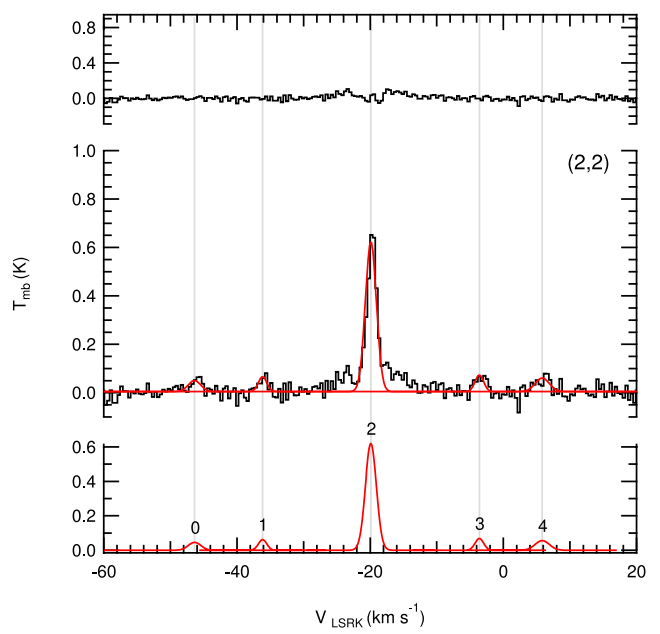

Figure 3. Left: spectrum of the $(1,1)$ metastable inversion line of ammonia centred on right ascension $10^{\mathrm{h}} 43^{\mathrm{m}} 20^{\mathrm{s}}$ and declination $-59^{\circ} 34^{\prime} 30^{\prime \prime}(\mathrm{J} 2000)$ with the Gaussian fits overlaid. The main peak is located at $-20 \mathrm{~km} \mathrm{~s}^{-1}$ with the satellite hyperfines located at the expected velocity offsets. Right: spectrum of the $(2,2)$ metastable inversion line of ammonia with the Gaussian fit overlaid. The vertical axis shows the brightness temperature in units of kelvin. The spectral axis is in units of $\mathrm{km} \mathrm{s}^{-1}$ defined in the local standard of rest kinematic frame. Note that hyperfines for the $(2,2)$ transitions are weakly present, and although it would be possible to use the $(2,2)$ optical depth to derive temperatures, this is not necessary if one assumes equivalent linewidths for the $(1,1)$ and $(2,2)$ transitions. The possibility of serving as a consistency check is prevented by the low-signal-to-noise ratio on the $(2,2)$ satellite hyperfines. 
The measurement errors on the optical depths were of the order 20 per cent. As the optical depth is the dominant term in a rotational temperature derivation, the errors for the resulting temperature maps are also of the order 20 per cent which is about 3-7 K depending on the pixel value. The temperature gradient increases towards the east for Car I-E and, similarly, to the west for Car I-W. This is consistent with the integrated intensity shifts in the emission map of the $(2,2)$ and $(3,3)$ levels (Fig. 2). As the temperature is proportional to the optical depth and the ratio of the $(1,1)$ and $(2,2)$ transitions, we might expect a similar global trend in optical depth, with peaks in optical depth. This expectation is at least globally present in the optical depth values presented in Table 2 which trace over the two velocity structures.

The Tr 14 cluster is about 3 arcmin (or $\sim 2.2$ pc) due directly east from the eastern extent of the emission maps. The far-ultraviolet (FUV) radiation field which escapes the stellar environment of this cluster is likely having a direct effect on the ammonia clouds. The cluster appears to be appreciably heating the gas in the eastern core, with kinetic temperature exceeding $50 \mathrm{~K}$ (Walmsley \& Ungerechts 1983), due to the significant emission of the $(3,3)$ metastable line. This is a significant increase from the cold temperatures usually associated with an IRDC (Fontani et al. 2012).

As there is clear detection of $(3,3)$ metastable line towards both clumps, the basic assumptions of relating $T_{\mathrm{R}}^{21}$ and $T_{k}$ are violated, and therefore, we do not derive kinetic temperatures. As such, we are unable to apply the approximations found in Rohlfs \& Wilson (2009) when analytically deriving column density.

\subsection{Comparisons to $\mathrm{CO}, \mathrm{IR}, \mathrm{H110} \alpha$ and maser sources}

The study of Brooks et al. (2003) is an ideal comparison, having studied the same region and derived equivalent parameters to this study. The fits to the $(1,1)$ and $(2,2)$ transitions which gave rise to the derived parameters are presented in Fig. 3 . The corresponding fit parameters are given in Table 3 and the derived parameters are listed in Section 3.3. In table 2 of Brooks et al. (2003), the equivalently derived parameters for $\mathrm{CO}$ are listed. In $\mathrm{CO}$ analysis the excitation temperature is a good approximation of the kinetic temperature in LTE. Brooks et al. (2003) derive an estimate of $31 \mathrm{~K}$ for emission in the -19 to $-21 \mathrm{~km} \mathrm{~s}^{-1}$ range. Although close to the rotational temperature $\left(T_{\mathrm{R} 21}\right)$ we derive for the Car I-W targeted observation $(21.9 \pm 3.6 \mathrm{~K})$, the existence of the $(3,3)$ emission line suggests significantly higher temperatures (Walmsley \& Ungerechts 1983). This suggests that the $\mathrm{CO}$ lines are saturated despite the spatially correlated $\mathrm{C}_{\text {II }} 158 \mu \mathrm{m}$ emission (implying photodissociation of the molecular species).

Our ammonia emission maps trace a similar ridge structure in fig. 5(a) presented in Brooks et al. (2003). The increased total linewidth to the north-east seems to correlate with the inner boundary of the Tr 14 front. Additionally, there is evidence of a depression in emission around right ascension $10^{\mathrm{h}} 43^{\mathrm{m}} 22^{\mathrm{s}}$ and declination $-59^{\circ} 35^{\prime} 00^{\prime \prime}$ that correlates with the location of the $\mathrm{C}_{\mathrm{II}} 158 \mu \mathrm{m}$ emission. Brooks describes this CS emission as tracing the interface between the east and west clouds. Furthermore, the kinematic structure is in agreement with their fig. 7; however, we do not detect the -18 to $-14 \mathrm{~km} \mathrm{~s}^{-1}$ and -13 to $-10 \mathrm{~km} \mathrm{~s}^{-1}$ structures. Overall it would appear that our observations are consistent with the morphology schematic presented in fig. 12 of their paper.

Scalise \& Braz (1980) have discovered a water maser in the vicinity of Car I dark cloud, approximately 44 arcsec east of Car I-E, with a systemic velocity of $-20 \mathrm{~km} \mathrm{~s}^{-1}$, whilst in addition, Caswell et al. (1989) discovered another along the line of sight of the Car
I-E ammonia emission peak with a systemic velocity $-17.5 \mathrm{~km} \mathrm{~s}^{-1}$. Although the later coincides with the peak emission in Car I-E, the associated velocity does not match exactly with the molecular cloud velocity range $\left(-21\right.$ to $\left.-25 \mathrm{~km} \mathrm{~s}^{-1}\right)$. Whilst it is highly likely that it lies behind the molecular cloud, higher resolution data would be needed to make definitive statements.

Tapia et al. $(2003,2006)$ have suggested that the stellar objects located at the interface are an embedded young stellar population. Despite the low resolution of the emission maps, comparison of the stellar and radio-continuum source positions to the Car I-W and Car I-E interface, along with the morphology proposed by Brooks et al. (2003), supports the conclusion that the young stellar objects are located on or just behind ionization fronts associated with Car I-W.

\section{CONCLUSION}

With the Tidbinbilla radio telescope, we observed for the first time the molecular emission of ammonia in the region associated with the $\operatorname{Tr} 14$ photodissocation region.

The first time mapping of this region has led to the derivation of optical depths, which are found to be in the range of $1<\tau_{(1,1, m)}<2$, and temperatures, with rotational temperatures typically around 20 $30 \mathrm{~K}$ and globally increasing towards the fringes. This is particularly evident in Car I-E, indicating its proximity to the $\operatorname{Tr} 14$ cluster relative to the Car I-W which is said to lie further behind the cluster. The kinematic structure of the ammonia emission was in general agreement with the morphology proposed in the literature, furthermore, supporting the conclusion that there is a young stellar population embedded in Car I-W.

The velocity dispersions were found to be broadened towards the expected ionization fronts. Detection of the $(3,3)$ metastable line suggests significantly higher kinetic temperatures than those derived with $\mathrm{CO}$ studies, implying that the $\mathrm{CO}$ emission is saturated despite the photodissociation caused by the Tr 14 FUV field for Car I-E and additionally for Car I-W the embedded young stellar cluster. The observations presented here highlight the potential of the Tidbinbilla radio telescope for sensitive large-scale ammonia mapping. However, further high-resolution wideband observations of the region would provide a unique opportunity for examining triggered star formation.

\section{ACKNOWLEDGEMENTS}

We acknowledge and thank the insightful comments and suggestions provided by the anonymous referee, which resulted in significant improvements to the presentation and conclusions of this work.

TY would like to thank the CSIRO Astronomy and Space Science division for the opportunity, initially through the summer vacation programme, thereafter through the ATNF graduate programme, to perform this research with the Tidbinbilla $70 \mathrm{~m}$ radio telescope. The staff at the Canberra Deep Space Communications Complex and the ATNF have his gratitude for their excellent hospitality. We would like to thank Tom Kuiper, Jill Rathbourne, Kate Brooks, Michael Burton and Cormac Purcell for their valuable insight, comments and discussion regarding the research contained within this paper.

\section{REFERENCES}

Batrla W., Wilson T. L., Ruf K., Bastien P., 1983, A\&A, 128, 279

Bergin E. A., Langer W. D., 1997, ApJ, 486, 316 
Brooks K. J., Whiteoak J. B., Storey J. W. V., 1998, Publ. Astron. Soc. Aust., 15,202

Brooks K. J., Storey J. W. V., Whiteoak J. B., 2001, MNRAS, 327, 46

Brooks K. J., Cox P., Schneider N., Storey J. W. V., Poglitsch A., Geis N., Bronfman L., 2003, A\&A, 412, 751

Caswell J. L., Batchelor R. A., Forster J. R., Wellington K. J., 1989, Aust. J. Phys., 42, 331

de Graauw T., Lidholm S., Fitton B., Beckman J., Israel F. P., Nieuwenhuijzen H., Vermue J., 1981, A\&A, 102, 257

de Marchi G. et al., 2011, AJ, 739, 15

Feinstein A., 1995, Rev. Mex. Astron. Astrofis. Ser. Conf., 2, 57

Fontani F., Giannetti A., Beltrn M. T., Dodson R., Rioja M., Brand J., Caselli P., Cesaroni R., 2012, MNRAS, 423, 2342

Gardner F. F., Milne D. K., Mezger P. G., Wilson T. L., 1970, A\&A, 7, 349

Giveon U., Sternberg A., Lutz D., Feuchtgruber H., Pauldrach A. W. A., 2002, ApJ, 566, 880

Greenhill L. J., Kondratko P. T., Lovell J. E. J., Kuiper T. B. H., Moran J. M., Jauncey D. L., Baines G. P., 2003, ApJ, 582, 11

Ho P. T. P., Martin R. N., Myers P. C., Barrett A. H., 1977, ApJ, 215, 29

Hur H., Sung H., Bessell M. S., 2012, AJ, 143, 10

Kuchar T. A., Clark F. O., 1997, ApJ, 488, 224

Matsakis D. N., Brandshaft D., Chui M. F., Cheung A. C., Yngvesson K. S., Cardiasmenos A. G., Shanley J. F., Ho P. T. P., 1977, ApJ, 214, L67

Pineda J., Caselli P., Goodman A. A., 2008, ApJ, 679, 481

Rathborne J. M., Burton M. G., Brooks K. J., Cohen M., Ashley M. C. B., Storey J. W. V., 2002, MNRAS, 331, 85

Rathborne J. M., Brooks K. J., Burton M. G., Cohen M., Bontemps S., 2004, A\&A, 418, 563

Rohlfs K., Wilson T., 2009, Tools of Radio Astronomy, 5th edn. SpringerVerlag, Berlin
Sault R., Teuben P., Wright M., 1995, in Shaw R., Payne H. E., J. H., eds, ASP Conf. Ser. Vol. 77, A Retrospective View of Miriad. Astron. Soc. Pac., San Francisco, p. 433

Scalise E., Braz M. A., 1980, A\&A, 85, 149

Smith N., Brooks K. J., 2008, in Reipurth B., ed., ASP Monograph Publications, Vol. 5, Handbook of Star Forming Regions: Vol. II The Southern Sky. Astron. Soc. Pac., San Francisco, p. 693

Smith N., Egan M. P., Carey S., Price S. D., Morse J. A., Price P. A., 2000, ApJ, 532, 145

Smith N., Brooks K. J., Koribalski B. S., Bally J., 2006, ApJ, 645, 41

Stahler S. W., Palla F., 2005, The Formation of Stars. Wiley, New York, p. 865

Stutzki J., Winnewisser G., 1985, A\&A, 148, 254

Tapia M., Roth M., Vázquez R. A., Feinstein A., 2003, MNRAS, 339, 44

Tapia M., Persi P., Bohigas J., Roth M., Gmez M., 2006, MNRAS, 367, 513

Townes C. H., Ho P. T., 1983, ARA\&A, 21, 239

Townes C. H., Schawlow A. L., 1955, Microwave Spectroscopy. McGrawHill, New York

Ungerechts H., Winnewisser G., Walmsley C. M., 1986, A\&A, 157, 207

Vazquez R. A., Baume G., Feinstein A., Prado P., 1996, A\&AS, 116, 75

Walmsley C. M., Ungerechts H., 1983, A\&A, 122, 164

Whiteoak J. B., Otrupcek R. E., 1984, Proc. Astron. Soc. Aust., 5, 552

Zhang X., Lee Y., Bolatto A., Stark A. A., 2001, ApJ, 553, 274

This paper has been typeset from a $\mathrm{T}_{\mathrm{E}} \mathrm{X} / \mathrm{LAT}_{\mathrm{E}} \mathrm{X}$ file prepared by the author. 